\title{
Educação em saúde e a importância da perspectiva transcultural da enfermagem no contexto dos ribeirinhos em um município do estado do Pará: um relato de experiência
}

Health education and the importance of the transcultural perspective of nursing in the context of riverside dwellers in a municipality in the state of Pará: an experience report

La educación para la salud y la importancia de la perspectiva transcultural de la enfermería en el contexto de los ribereños de un municipio del estado de Pará: un relato de experiencia

Jucilene Luz Neves ${ }^{1}$, Rogéria de Sousa Rodrigues ${ }^{1}$, Ana Carolina Marinho Pinheiro ${ }^{1}$, Iris Marques Rodrigues ${ }^{1}$, Helber Freitas Tavares ${ }^{1}$, Vanessa Stephany Souza da Silva ${ }^{1}$, Thaís Neves de Souza ${ }^{1}$, Gleice de Araujo Steinheuser ${ }^{2}$, Geice Kelly Costa Soares Garcia ${ }^{1}$, Leonardo Fabiano Sousa Malcher ${ }^{1 *}$.

\section{RESUMO}

Objetivo: Relatar a experiência vivenciada por acadêmicos de enfermagem, debatendo sobre a perspectiva da diversidade cultural, e a importância da enfermagem transcultural. Relato de experiência: A atividade foi desenvolvida no dia 09 de outubro de 2018, em uma instituição de ensino de nível técnico profissionalizante, localizado em um município do estado do Pará. Teve como ponto de partida a distribuição de uma enquete auto-avaliativa na qual tentou-se diagnosticar o conhecimento prévio dos alunos acerca do tema, após esse primeiro momento foi feita a apresentação da cartilha que retrata a assistência de enfermagem ao povo ribeirinho. Num terceiro momento, iniciamos reflexões com os alunos acerca da importância deste conhecimento tanto para os discentes da faculdade proponente da atividade quanto para os alunos que foram alvo da intervenção. Considerações finais: As ações lúdicas voltadas ao debate acerca da diversidade cultural bem como sobre a enfermagem transcultural se fazem extremamente necessárias para que se capacite os profissionais de enfermagem para prestar cuidados culturalmente competentes e que atenda as necessidades englobando o modo de vida dos usuários.

Palavras-chave: Cultura, Diversidade cultural, Enfermagem transcultural.

\section{ABSTRACT}

Objective: To report the experience lived by nursing students, discussing the perspective of cultural diversity, and the importance of cross-cultural nursing. Experience report: The activity was developed on October 9 , 2018, in an educational institution at a technical professional level, located in a municipality in the state of Pará. Its starting point was the distribution of a self-evaluation survey in which an attempt was made to diagnose the students' prior knowledge on the subject, after this first moment the booklet that portrays nursing care for the riverside people was presented. In a third moment, we started reflections with the students about the importance of this knowledge both for the students of the faculty proposing the activity and for the students who were the target of the intervention. Final considerations: Playful actions aimed at the debate on cultural diversity as well as on transcultural nursing are extremely necessary to enable nursing professionals to provide culturally competent care and to meet the needs encompassing the users' way of life.

Keyword: Culture, Cultural diversity, Transcultural nursing.

${ }^{1}$ Centro Universitário Metropolitano da Amazônia (UNIFAMAZ), Belém - PA.

*E-mail: leonardo malcher@yahoo.com.br

2 Universidade Da Amazônia (UNAMA), Belém - PA. 


\section{RESUMEN}

Objetivo: Informar la experiencia vivida por los estudiantes de enfermería, discutiendo la perspectiva de la diversidad cultural y la importancia de la enfermería transcultural. Informe de experiencia: La actividad se desarrolló el 9 de octubre de 2018, en una institución educativa a nivel técnico profesional, ubicada en un municipio del estado de Pará. Su punto de partida fue la distribución de una encuesta de autoevaluación en la que se intentó realizado para diagnosticar los conocimientos previos de los estudiantes sobre el tema, luego de este primer momento se presentó el folleto que retrata los cuidados de enfermería para los pobladores ribereños. En un tercer momento, iniciamos reflexiones con los estudiantes sobre la importancia de este conocimiento tanto para los estudiantes de la facultad que proponen la actividad como para los estudiantes que fueron el objetivo de la intervención. Consideraciones finales: Las acciones lúdicas dirigidas al debate sobre la diversidad cultural así como sobre la enfermería transcultural son sumamente necesarias para que los profesionales de enfermería brinden cuidados culturalmente competentes y satisfagan las necesidades que abarcan el modo de vida de los usuarios.

Palabra clave: Cultura, Diversidad cultural, Enfermería transcultural.

\section{INTRODUÇÃO}

A diversidade cultural envolve diferentes contextos que são fundamentais ao pensarmos a realidade da saúde, tais como a religiosidade, raça, sexualidade, tradições, valores de cada grupo e de suas relações sociais. Para a antropologia, diversidade cultural representa todos os valores e práticas que mostram os padrões desenvolvidos e reproduzidos pelos próprios seres humanos em sua cultura, e é neste sentido que refletirmos que a cultura de um povo, sua história, seu espaço e visão de mundo em determinado contexto sociocultural é fundamental para a nossa reflexão aqui (AMARANTE P e COSTA AM, 2012).

A cidade em que se realiza o estudo possui uma vasta diversidade cultural, a qual é possível perceber a partir de influências que vão de grupos indígenas a comunidades ribeirinhas, cada um com singularidades, especificidades, e visões de mundo sobre todos os aspectos de sua vida cotidiana, de sua história, e também sobre o que vem a ser entendido por saúde (NUNES TG, 2016).

Os ribeirinhos moradores das florestas e das margens dos rios possuem um vasto conhecimento do ambiente local e criam a partir deste contexto uma série de narrativas, voltadas principalmente para a relação homem/natureza (LIRA TM e CHAVES MPSR, 2016). Esses povos sobrevivem dos insumos da floresta, da pesca e artesanato, possuem uma peculiaridade quanto à perspectiva do cuidar fazendo uso de ervas medicinais além de outros conhecimentos acerca da saúde repassando seus hábitos e seus valores culturais de geração em geração (SILVA HPF, et al., 2016).

A cultura de um povo é pautada nos seus valores, símbolos e significados dados à sua vida e ao seu meio social em que vivem e está relacionada diretamente à configuração de sua identidade que deve ser respeitada e valorizada inclusive no que diz respeito à saúde. Levando em consideração este dado é que devemos discutir como fundamental a perspectiva transcultural, que, na enfermagem visa buscar conhecer a diversidade cultural e flexibilizar o olhar diante das mais variadas culturas, estabelecendo uma assistência benéfica pautada no respeito à diversidade (GERHARDT TE, 2019).

Analisar e perceber a influência das tradições e costumes dos povos ribeirinhos acerca dos seus itinerários terapêuticos e cuidados com a saúde apresenta-se como uma necessidade de conhecimento dos profissionais de saúde, sobretudo dos profissionais da enfermagem acerca dos costumes e tradições de um povo, para assim promover uma assistência de enfermagem com o máximo de excelência. Pelo fato da assistência ser centrada no usuário e no território em que ele habita, há um desafio para a gestão em saúde, onde deve-se conhecer as características desses sujeito bem como o que determina as suas buscas pelo cuidado, para além de saber se a busca acontece, é primordial saber em que momento acontece e quais seus significados para os sujeitos (CABRAL ALLV, et al., 2011). 
Conhecer a realidade científica e a perspectiva tradicional, os conhecimentos científicos e empíricos como não excludentes proporcionará uma melhor compreensão da realidade para que se trace um melhor plano terapêutico para cada indivíduo e grupo no contexto cultural local. Sendo assim, a formação de enfermeiros que tragam consigo a perspectiva transcultural é de suma importância para o êxito no cuidado de enfermagem, uma vez que o processo de saúde e doença também é influenciado pelo âmbito sociocultural (NUNES TG, 2016).

Partindo desta perspectiva e tendo a disseminação de conhecimento que fundamenta-se na importância da Promoção e Educação em Saúde, tema tão importante para a formação e a prática do (a) profissional da enfermagem, entende-se que educação em saúde, é uma das competências do enfermeiro, pois reporta práticas de conhecimento no seu campo de atuação, além de realizar diversas ações em conjunto com outras organizações e instituições permitindo a construção de novas habilidades e contribuindo para a autonomia do cuidado individual e coletivo (FALKENBERG MB, et al., 2013).

Práticas inovadoras e transformadoras foram consideradas item necessários para formação do enfermeiro, visto que, a parti delas tornou-se capaz de desenvolver pensamento crítico para ações voltadas para promoção e prevenção da saúde da população, articulando teoria e prática com intuito de modificar os fatores determinantes a fim de promover uma melhor qualidade de vida para cada grupo respeitando suas singularidades (COLOMÉ JS e OLIVEIRA DLLC, 2012).

Logo o objetivo deste estudo foi relatar a experiência vivenciada por acadêmicos de enfermagem, debatendo sobre a perspectiva da diversidade cultural, e a importância da enfermagem transcultural.

\section{RELATO DE EXPERIÊNCIA}

A experiência aqui relatada foi realizada por discentes graduandos do $1^{\circ}$ e $2^{\circ}$ semestres do Curso de Bacharelado em Enfermagem de um Centro Universitário localizado em um município do estado do Pará, e faz parte da conclusão das disciplinas de ambos os semestres como prática curricular exigida no curso de Enfermagem e teve orientação e supervisão de docente da própria instituição. Após a construção do grupo de discentes foi feita a escolha do tema a ser trabalhado que, no caso, foi trabalhar o tema da transculturalidade relacionando-o especificamente ao contexto amazônico e na perspectiva dos ribeirinhos. A partir da seleção do tema, o aspecto da educação e conscientização sobre o tema foi o foco central.

A execução da experiência teve início com a confecção de uma cartilha elaborada pelos próprios discentes e direcionadas para a educação/informação acerca do tema junto aos alunos de nível técnico de enfermagem, público-alvo da nossa experiência. A experiência foi projetada em algumas etapas que foram desenvolvidas, após prévio contato com a coordenação da instituição, expondo-lhe os objetivos, a importância e a contribuição para os futuros profissionais de enfermagem, no dia 09 de outubro de 2018 , em uma instituição de ensino de nível técnico profissionalizante, localizado em um município do estado do Pará. O público-alvo com o qual desenvolveu-se a atividade foi o de 14 alunos Curso Técnico de Enfermagem da instituição, sendo 13 mulheres e 1 homem com faixa etária variando de 18 a 45 anos.

Num primeiro momento, os discentes aplicaram uma enquete auto avaliativa que teve como intuito diagnosticar o conhecimento prévio dos alunos acerca do tema. Num segundo momento, foi feita a apresentação da cartilha que retratava a importância da diversidade cultural, da Enfermagem Transcultural e a assistência de enfermagem ao povo ribeirinho.

Num terceiro momento, iniciamos reflexões com os alunos acerca da importância deste conhecimento tanto para os discentes da faculdade proponente da atividade quanto para os alunos que foram alvo da intervenção, buscando evidenciar que em todos os níveis de conhecimento a temática é fundamental. Além destes três momentos, foram realizadas três dinâmicas, pelas quais, a partir da interação com os alunos, foram feitas perguntas abertas para que todos se expressassem e participassem de forma mais dinâmica e para uma melhor socialização entre os participantes.

Por fim, foi realizada uma roda de conversa para discutir o processo que envolve a diversidade cultural com a distribuição de uma caixinha contendo perguntas, onde os participantes permaneceram sentados em 
círculo e, ao ritmo de uma música passavam a caixinha de mão em mão até que a música parava e o aluno que estivesse com a caixinha nas mãos retirava uma pergunta respondendo-a e interagindo com o grupo, atividade que fora realizada cinco vezes. Ao término das atividades, foi recolhida a enquete que havia sido entregue no início para que pudesse ser mensurado o alcance das atividades executadas.

Ao término de todos os momentos de interação e a realização de todas as atividades, pode-se afirmar que houve algumas dificuldades, como: curto intervalo de tempo para realizar a atividade com os alunos, a ausência de recursos eletrônicos que auxiliassem a execução das ações o que tornou inviável o cumprimento da atividade como fora planejada havendo a necessidade de adequações rápidas para suprir a demanda da apresentação eletrônica, como ocorreu quando a apresentação da cartilha se deu em uma roda de conversa com os alunos.

Notou-se que há uma grande necessidade, por parte dos alunos, de compreender e lidar com diversos hábitos, aspectos e comportamentos culturais como ponto importante para a sua prática de enfermagem. Contudo, pode-se afirmar que os alunos foram muito participativos ao discutirem a construção da enfermagem no processo da diversidade cultural, envolvendo-se por meio do diálogo e buscando ampliar conhecimentos de acordo com as peculiaridades culturais propostas durante a ação.

Ao curso do processo todos os alunos demostraram interesse, no entanto, um grupo de alunos apresentou uma melhor assimilação acerca da temática abordada compartilhando com os demais suas experiências sobre a maneira de lidar com a diferença cultural no cotidiano, considerando a cultura ribeirinha. Em contrapartida, percebeu-se que os demais alunos não detinham conhecimento sobre o tema exposto embora se identificassem com o mesmo de forma indireta.

As atividades desenvolvidas destinaram-se a obtenção de conhecimento do aluno técnico de enfermagem sobre a diversidade cultural em um município do estado do Pará, em específico a cultura dos ribeirinhos das ilhas que constituem este município, e como a enfermagem se insere nesse contexto. Os acadêmicos de enfermagem durante as ações desenvolvidas contribuíram com o conhecimento, acerca da diversidade cultural e com a prevenção de possíveis dificuldades dos profissionais de enfermagem frente às diversas adaptações e necessidades culturais dos pacientes e seus familiares.

\section{DISCUSSÃO}

A região amazônica passou por vários processos de miscigenação e colonização, sendo assim, é ocupada em sua maioria por diversos grupos étnicos e povos tradicionais. $O$ resultado desses processos é evidente a partir de alguns aspectos da vida cotidiana do homem amazônico o que se torna evidente nas manifestações socioculturais, nas narrativas populares, lendas, na educação e reprodução social dos costumes e nos hábitos da sua vida cotidiana. A produção dentro das comunidades ribeirinhas é pautada na relação homem-natureza, elas fazem uso dos seus saberes tradicionais os quais são repassados de geração em geração, estabelecendo uma relação entre o ambiente e os recursos locais, como a influência da lua no corte de madeira, no roçado, pesca entre outros (LIRA TM e CHAVES MPSR, 2016).

De modo geral, as comunidades ribeirinhas são constituídas em suma por trabalhadores que sobrevivem do extrativismo de frutos como o açaí, cacau, buriti, mas também da confecção de artesanato e sobretudo da pesca. Tal população possui um estilo de vida próprio, o que para muitos pode ser considerado de forma bastante etnocêntrica como "atrasado" nada mais é do que a identidade de um povo, portanto, pensar políticas públicas que atendam as particularidades e peculiaridades desses povos pode ser um grande desafio para 0 poder público (SANTOS CRG et al., 2012).

O uso medicinal de diversas plantas por essas comunidades para o tratamento de várias doenças, que muitas vezes são também reconhecidas e estudada pela medicina dita oficial é extremamente comum. Os tratamentos e todo um itinerário próprio para determinado evento de adoecimento são empregadas também para o tratamento de doenças que se originam nas crenças populares como, por exemplo, o quebranto e 0 mau olhado. Além disso, as comunidades têm preferência principalmente pelos tratamentos que envolvem em seu arsenal as plantas para a preparação dos chás que estão disponíveis com facilidade e abundância nas comunidades (MARQUES WPG et al., 2020). 
Este dado relaciona-se diretamente como fato de que a enfermagem é uma profissão que fornece cuidados ao ser humano nos mais diversos contextos sociais e culturais onde se processam diferentes formas e estratégias de cuidado em saúde. Diante disso, é dever da enfermagem em todos os seus níveis de formação, buscar conhecer teorias que subsidiem sua prática de forma mais resolutiva e eficiente (HENCKMAIER L, 2014).

A experiência aqui relatada buscou intervir de modo a perceber que, dada a enorme diversidade cultural existente, o cuidado a pacientes de qualquer parte do mundo é uma realidade que traz inúmeros desafios para a enfermagem. Portanto, é necessário que os cuidados realizados sejam culturalmente competentes, contribuindo assim para a manutenção da saúde, respeitando a singularidade dos indivíduos, família, grupos e comunidades. Entender o outro é ir de encontro com a singularidade que engloba inclusive a cultural, tal ação facilita o vínculo para a construção de uma relação terapêutica que possibilite cuidados de enfermagem culturalmente adequados (CORDEIRO RASB, 2016).

Esta reflexão, pelo que o grupo entendeu e buscou disseminar, é fundamental para todos os profissionais da área da saúde, e no caso exposto aqui, na enfermagem, não apenas para discentes do nível superior que se interessam pela temática, mas para aqueles profissionais da área que estão na sua formação profissional em nível intermediário (CORDEIRO RASB, 2016).

Desse modo os profissionais de enfermagem e os usuários devem estabelecer diálogos fundamentados na escuta terapêutica, respeitando e valorizando as experiências individuais, coletivas e a história de vida desses usuários (CEVVERA DPP, et al., 2011). Com o passar dos anos os ribeirinhos adquiriram formas de manejar a saúde que são tradicionais e estão relacionadas com o contexto regional e cultural, os quais envolvem uso de chás, benzimentos e uso de ervas nativas, tais práticas evidenciam a maneira própria com que os ribeirinhos concebem o processo saúde-doença (BÔAS LMSV e OLIVEIRA DC, 2017).

Vale ressaltar que o modelo biomédico que vigora torna difícil a assistência de forma integral ao usuário este, no entanto busca cada vez mais alternativas de tratamento para a manutenção da saúde, portanto é de suma importância que o profissional de enfermagem conheça os princípios científicos das plantas e intercale o saber popular com o científico, dado o acesso frequente dessas populações a esses elementos (FERREIRA $\mathrm{ET}$, et al., 2019).

A ação com construção da tecnologia educativa permitiu a aproximação dos discentes no que se refere as práticas de saúde dos povos ribeirinhos. Assim, a educação em saúde pode ser compreendida como um processo teórico-prático que engloba saberes científicos, populares e do senso comum, com a finalidade de desenvolver a visão crítica no que tange a produção dos cuidados em saúde (RAMOS CFV, et al., 2017).

As ações lúdicas voltadas ao debate acerca da diversidade cultural bem como sobre a enfermagem transcultural se fazem extremamente necessárias para que se capacite os profissionais de enfermagem para prestar cuidados culturalmente competentes e que atendam às necessidades englobando o modo de vida dos usuários de forma que haja respeito ás vivências, experiências e todo conhecimento popular adquirido, garantindo assim, que estes se sintam acolhidos e se tenha conforto e espontaneidade na relação paciente e enfermeiro.

\section{REFERÊNCIAS}

1. AMARANTE P, COSTA AM. Diversidade cultural e saúde. CEBES - Centro brasileiro de estudos de saúde, 2012.

2. BÔAS LMSV, OLIVEIRA DC. Diferentes saberes implicados no cuidado de saúde ribeirinho: análise teórica. Revista Presença Geográfica, 2017.

3. CABRAL ALLV, et al. Itinerários terapêuticos: o estado da arte da produção científica no Brasil. Ciência \& Saúde Coletiva, 2011; 16(11): 4433-4442.

4. CERVERA DPP, et al. Educação em saúde: percepção dos enfermeiros da atenção básica em Uberaba (MG). Ciência \& Saúde Coletiva, 2011; 16(Supl. 1): 1547-1554. 
5. COLOMÉ JS, OLIVEIRA DLLC. Educação em saúde: por quem e para quem? A visão de estudantes de graduação em enfermagem. Texto \& Contexto - Enfermagem [online], 2012; 21(1).

6. CORDEIRO RASB. O Cuidar Culturalmente Competente à Criança, ao Jovem e Família com Doença Crônica. Escola Superior de Enfermagem de Lisboa, 2016.

7. FALKENBERG M B, et al. Educação em saúde e educação na saúde: conceitos e implicações para a saúde coletiva. Ciência \& Saúde Coletiva, 2014; 19: 847-852.

8. FERREIRA ET, et al. Utilização de plantas medicinais e fitoterápicos: uma revisão integrativa sobre a atuação do enfermeiro. Braz. J. Hea. Rev., Curitiba, 2019; 2(3): 1511-1523.

9. GERHARDT TE. Cultura e cuidado: dilemas e desafios do ensino da antropologia na graduação em Saúde Coletiva. Saúde e Sociedade, 2019; 28: 38-52.

10. HENCKEMAIER L, et al. Cuidado Transcultural de Leininger na Perspectiva dos Programas de PósGraduação em enfermagem: revisão integrativa. Ciência \& Saúde, 2014; 7(2): 85-91.

11.LIRA TM, CHAVES MPSR. Comunidades ribeirinhas na Amazônia: Organização sociocultural e política. Interações, 2016; 6(17): 66-76.

12.MARQUES WPG, et al. Plantas medicinais usadas por comunidades ribeirinhas do Estuário Amazônico. Brazilian Journal of Development, Curitiba, 2020; 6(10): 74242-74261.

13.NUNES TG. Mobilização local: ribeirinhos e a luta pela melhoria de vida na área insular de Belém/PA. Emblemas - revista da unidade acadêmica especial de história e ciências sociais - UFG/CAC, 2016.

14.RAMOS CFV, et al. Práticas educativas: pesquisa-ação com enfermeiros da Estratégia de Saúde da Família. Revista Brasileira de Enfermagem, 2018; 71: 1144-1151.

15.SANTOS CRG, et al. Ribeirinhos da Amazônia: modo de vida e relação com a natureza. V Encontro da Rede de Estudos Rurais, 2012.

16.SILVA HPF, et al. Doença e cura em uma perspectiva sócio-cultural. Razón y Palabra, 2016; 20(95): 337346. 